Swarthmore College

Works

8-1-2012

\title{
Spectroscopic Observation Of Simultaneous Bi-Directional Reconnection Outflows In A Laboratory Plasma
}

\author{
Michael R. Brown \\ Swarthmore College, doc@swarthmore.edu \\ C. D. Cothran \\ Timothy G. Gray , '01 \\ C. E. Myers \\ E. V. Belova
}

Follow this and additional works at: https://works.swarthmore.edu/fac-physics

Part of the Physics Commons

Let us know how access to these works benefits you

\section{Recommended Citation}

Michael R. Brown; C. D. Cothran; Timothy G. Gray , '01; C. E. Myers; and E. V. Belova. (2012).

"Spectroscopic Observation Of Simultaneous Bi-Directional Reconnection Outflows In A Laboratory

Plasma". Physics Of Plasmas. Volume 19, Issue 8. DOI: 10.1063/1.4747345

https://works.swarthmore.edu/fac-physics/102

This work is brought to you for free by Swarthmore College Libraries' Works. It has been accepted for inclusion in Physics \& Astronomy Faculty Works by an authorized administrator of Works. For more information, please contact myworks@swarthmore.edu. 


\title{
AIP $\left.\right|_{\text {Physics of }} ^{\text {Plo }}$ \\ Plasmas
}

\section{Spectroscopic observation of simultaneous bi-directional reconnection outflows in a laboratory plasma}

\author{
M. R. Brown, C. D. Cothran, T. Gray, C. E. Myers, and E. V. Belova
}

Citation: Physics of Plasmas (1994-present) 19, 080704 (2012); doi: 10.1063/1.4747345

View online: http://dx.doi.org/10.1063/1.4747345

View Table of Contents: http://scitation.aip.org/content/aip/journal/pop/19/8?ver=pdfcov

Published by the AIP Publishing

Articles you may be interested in

Experimental observation of 3-D, impulsive reconnection events in a laboratory plasma

Phys. Plasmas 21, 012109 (2014); 10.1063/1.4862039

Excitation and propagation of electromagnetic fluctuations with ion-cyclotron range of frequency in magnetic reconnection laboratory experiment

Phys. Plasmas 20, 061209 (2013); 10.1063/1.4811469

Magnetohydrodynamic structure of a plasmoid in fast reconnection in low-beta plasmas

Phys. Plasmas 18, 022105 (2011); 10.1063/1.3554655

Experimental study of the Hall effect and electron diffusion region during magnetic reconnection in a laboratory plasma

Phys. Plasmas 15, 082113 (2008); 10.1063/1.2936269

The reconnection of magnetic fields between plasmas with different densities: Scaling relations

Phys. Plasmas 14, 102309 (2007); 10.1063/1.2772619

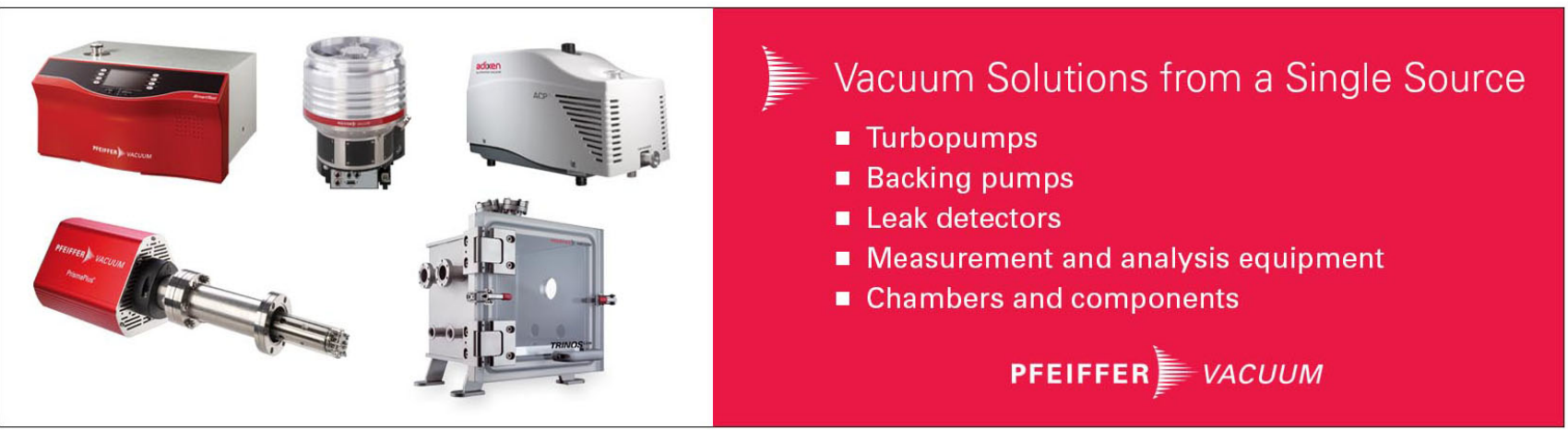




\title{
Spectroscopic observation of simultaneous bi-directional reconnection outflows in a laboratory plasma
}

\author{
M. R. Brown, ${ }^{1, a)}$ C. D. Cothran, ${ }^{1}$ T. Gray, ${ }^{1}$ C. E. Myers,${ }^{2}$ and E. V. Belova ${ }^{2}$ \\ ${ }^{1}$ Department of Physics and Astronomy, Center for Magnetic Self Organization, Swarthmore College, \\ Swarthmore, Pennsylvania 19081-1397, USA \\ ${ }^{2}$ Princeton Plasma Physics Laboratory, Princeton, New Jersey 08543, USA
}

(Received 13 June 2012; accepted 6 August 2012; published online 27 August 2012)

\begin{abstract}
We report a precise, direct spectroscopic measurement of simultaneous bi-directional outflows from a reconnection event in a laboratory plasma. Outflow speeds are as Alfvénic and Abel analysis shows that the outflows are generated in the plasma core. A Sweet-Parker like analysis of outflow speed coupled with external measurements of reconnection electric field and assumption of Spitzer resistivity predict an aspect ratio of the reconnection layer and reconnection rate that are close to that measured in the experiment and in simulations. However, this analysis underestimates the absolute scale of the layer, indicating other than 2D resistive physics is at play. (C) 2012 American Institute of Physics. [http://dx.doi.org/10.1063/1.4747345]
\end{abstract}

Magnetic reconnection is a process involving local annihilation of magnetic flux and global rearrangement of magnetic field lines. The process of reconnection necessarily results in a loss of magnetic energy with a subsequent increase in energy of ions and electrons in the plasma (flows and heating). Reconnection is invoked in the solar corona as a possible mechanism for coronal heating ${ }^{1}$ and coronal mass ejections. $^{2}$ In this letter, we report a precise, direct laboratory measurement of simultaneous bi-directional outflows from a reconnection volume at a substantial fraction of the Alfvén speed (the characteristic speed of magnetohydrodynamics or MHD, $\left.v_{A}=B / \sqrt{\mu_{0} \rho}\right)$. The measurement is performed with a novel high-spectral and high-temporal resolution ion Doppler spectrometer. The bi-directional outflows are correlated spatially with the formation of a complex three dimensional reconnection volume and temporally with the rapid annihilation of magnetic flux.

Reconnection outflows have been reported in several physical systems spanning a vast range of parameters. In a typical scenario, parcels of magnetized plasma with oppositely directed field lines are convected into a reconnection volume at a relatively slow inflow speed, $v_{i n}$, and ejected at an outflow speed, $v_{\text {out }}$, which is often nearly Alfvénic. The Sweet-Parker model ${ }^{1}$ predicts that in steady state, the inflow speed and the layer thickness $\delta$ adjust themselves such that magnetic flux is annihilated in the layer at the rate at which plasma is exhausted out the ends. The model prediction is that the normalized reconnection rate $v_{\text {in }} / v_{\text {out }}$ equals the aspect ratio of the layer $\delta / L$, and the scaling is such that $v_{\text {in }} / v_{\text {out }}=\delta / L=1 / \sqrt{S}$, where $\mathrm{S}$ is the Lundquist number using the large scale width of the layer $L$. This suggests a technique to predict internal structure, perhaps below detector resolution, using external measurements such as $v_{\text {in }}$ and $v_{\text {out }}$.

Bi-directional jets emerging from two sides of a reconnection volume have been observed spectroscopically in the solar chromosphere with the ultraviolet spectrometer, solar

\footnotetext{
${ }^{\text {a)} E l e c t r o n i c ~ a d d r e s s: ~ d o c @ s w a r t h m o r e . e d u . ~}$
}

ultraviolet measurements of emitted radiation (SUMER) instrument, on the satellite Solar and Heliospheric Observatory $(\mathrm{SOHO}){ }^{3,4}$ In these remarkable measurements, dynamics of a $S i_{I V}$ impurity line show simultaneous red- and blue-shifts corresponding to flows up to $100 \mathrm{~km} / \mathrm{s}$ and localized to a small portion (about $10^{-4}$ ) of the solar surface area and about $10000 \mathrm{~km}$ in extent, reaching into the low corona. Higher in the solar corona, reconnection-driven outflows known as X-ray jets have been observed with several instruments. ${ }^{5-7}$ In the solar wind and magnetosphere, there have been several reports of nearly simultaneous bi-directional reconnection outflows. ${ }^{8}$ Bi-directional jets have also been photographed in laser-generated plasmas at the surface of a solid target $^{9,10}$ and single outflows have been measured spectroscopically in laboratory experiments. ${ }^{1,12}$ Reconnection outflows have been observed in laboratory experiments for decades using in situ electrostatic probes, ${ }^{13}$ but typically both outflows are not measured simultaneously (within a dynamical time), and typically not using high-resolution, nonperturbative spectroscopy.

The Swarthmore Spheromak Experiment (SSX) is a flexible facility dedicated to the study of magnetic reconnection through the merger of force-free loops of magnetized hydrogen plasma called spheromaks. ${ }^{14,15}$ We observe simultaneous bi-directional outflow jets with outflow speeds up to $\pm 40 \mathrm{~km} / \mathrm{s}$ using a high resolution ion Doppler spectrometer. The outflow is temporally and spatially correlated with magnetic reconnection events. SSX plasmas have electron density up to $10^{21} \mathrm{~m}^{-3}$, temperatures $T_{e} \sim T_{i} \cong 20 \mathrm{eV}$, and typical magnetic fields of $0.1 \mathrm{~T}$. These parameters give an external Alfvén speed of $70 \mathrm{~km} / \mathrm{s}$. The plasma is fully ionized and fully magnetized $\rho_{i} \ll R$, where $R=0.2 \mathrm{~m}, L=0.6 \mathrm{~m}$ is the outer flux conserving boundary of the plasma (defined by a cylindrical copper wall). The large-scale Lundquist number $S$, the ratio of the resistive magnetic diffusion time $\tau_{R}$ to the Alfvén transit time $\tau_{A}$, is large for $\mathrm{SSX}, S \cong 1000$. Accordingly, the global structure of SSX spheromaks is fully in the MHD regime $\left(S \gg 1, \rho_{i} \ll R\right)$. 
We generate and merge two loops of magnetized plasma containing oppositely directed magnetic fields in order to produce optimal conditions for magnetic reconnection at the midplane of our machine. The two plasma loops are convected at an inflow speed $v_{\text {in }}$ into a highly evacuated, low magnetic field volume. Plasma is formed at $t=0$ in separate magnetized plasma guns. Merging begins at the midplane at about $30 \mu$ s. In Figure 1(a), we depict a schematic representation of the SSX with two plasma loops merging. In Figure 1(b), we show data from a three dimensional magnetic probe array (600 individual detectors) ${ }^{16}$ of the reconnection layer. The data depicted are from earlier experiments with a $0.5 \mathrm{~m}$ flux conserver, but the structure in our $0.4 \mathrm{~m}$ flux conserver is similar. The line of sight for our ion Doppler spectrometer is through this reconnection layer.

We have recently implemented an ion Doppler spectroscopy (IDS) diagnostic with both high spectral and temporal resolution. ${ }^{17}$ Velocity resolution is about $7 \mathrm{~km} / \mathrm{s}$, corresponding to approximately $0.1 v_{A}$. Line shapes are recorded at $1 \mu$ s cadence (the dynamical time) with a 32 channel photomultiplier tube array $(1 \mathrm{~mm}$ pitch). Light is recorded from plasma along a chord with about $1.5 \mathrm{~cm}$ spatial resolution. In Figure 2, we show a typical line shape from $C_{I I I}$ emission measured during a single reconnection event at $40 \mu$ s after plasma formation. At our densities, the collision time between $C_{I I I}$ ions and protons is always less than $1 \mu \mathrm{s}$, so these measurements accurately track the dynamics of the majority ions. Since the reconnection zone is localized and dynamic, we capture simultaneous bi-directional jets such as this on only $10 \%$ of SSX merging shots. Reconnection jets could be bursty in time, but also a slight axial shift of $1.5 \mathrm{~cm}$ moves the layer and jet out of the IDS line-of-sight. The chord for this sequence is across the full $0.4 \mathrm{~m}$ diameter at the midplane, as shown in Figure 1. Gaussian fits for the line shapes are displayed as well as the raw data from each channel. Motion both radially in at $38.1 \pm 0.3 \mathrm{~km} / \mathrm{s}$ (redshift) and radially out at $30.4 \pm 0.3 \mathrm{~km} / \mathrm{s}$ (blueshift) is evident and can be measured to within a few percent. The ion temperature of each parcel is about $20 \mathrm{eV}$. It is interesting to note that at this time (and for several $\mu$ s after) and along this chord, virtually all the plasma is flowing either radially in or out, i.e., there seems to be no plasma at rest. This is a common feature of SSX merging shots.

We can measure line shapes along a number of chords across the device at the midplane in order to verify that emission is coming from reconnection at the plasma core. In Figure 3, we plot the total emissivity of the $C_{I I I}$ line as a function of impact parameter for 10 different chords. The intensity of the light is integrated across the entire line and 10 shots are averaged at each chord. Data are also averaged into $5 \mu$ s time bins to smooth shot-to-shot variability (black curve with data and error bars). Since jets are observed only $10 \%$ of the shots, and only for a few $\mu$ s for each event, the non-axisymmetric character of the jets affects the Abel inversion very little. A cubic spline is then fit to the data. Two matched splines are used, one that vanishes at the wall and another with vanishing derivative at $r=0$. The spline fit is Abel-inverted to generate a plot of the emissivity as a function of radius (blue curve with blue band for error bars). Finally, the Abel inverted data can be used to re-construct the emissivity profile as a function of impact parameter (red smooth curve). If the inversion is correct, then the reconstruction should overlie the original data.

Note that at the time depicted ( $t=40 \mu \mathrm{s})$, the $C_{I I I}$ emission primarily comes from the core of the plasma (around $r=10-15 \mathrm{~cm}$ ) where we expect the most reconnection activity and the hottest plasma. Magnetic data at the midplane show that the center of the reconnection zone is at $r=14 \mathrm{~cm}$ (Ref. 18), while the peak emission is from $r=12 \mathrm{~cm}$. From magnetic measurements, we know that at this time, reconnection dynamics have commenced but the merged object is still nearly axisymmetric. Abel inversion requires that the data be axisymmetric. Later in time, our plasma becomes non-axisymmetric and Abel inversion is not possible. This measurement indicates that $C_{I I I}$ is a good tracer of bulk properties so we are not just measuring edge effects. We find that emissivity is highest immediately after the spheromak merging process (around $40-50 \mu \mathrm{s}$ ) and low during early formation and late decay.

A Sweet-Parker-like analysis allows us to use external measurements to infer the internal aspect ratio of the layer $\delta / L$. This is helpful experimentally if internal probing is not possible or desired. In some scenarios, direct measurement of $v_{\text {in }} / v_{\text {out }}$ could be used..$^{2}$ In the case of SSX, our most accurate external measurements are of (1) the reconnection electric field $E=v \times B=200 \mathrm{~V} / \mathrm{m}$ determined from the slope of externally measured magnetic flux vs. time $\phi(t)$ in Figure 4(a) of Cothran et al. ${ }^{18}$ between 40 and $50 \mu$ s, (2) the lineaveraged density $n_{e}=10^{15} \mathrm{~cm}^{-3}$ from Figure 7(b) of Cothran et al., ${ }^{18}$ and (3) the outflow speed, $v_{\text {out }}=30 \mathrm{~km} / \mathrm{s}$ reported here from the same set of experiments.

In order to perform the analysis, we assume (1) axisymmetry so we can extract a reconnection electric field from a non-local flux measurement, (2) steady state so $E$ can be a)

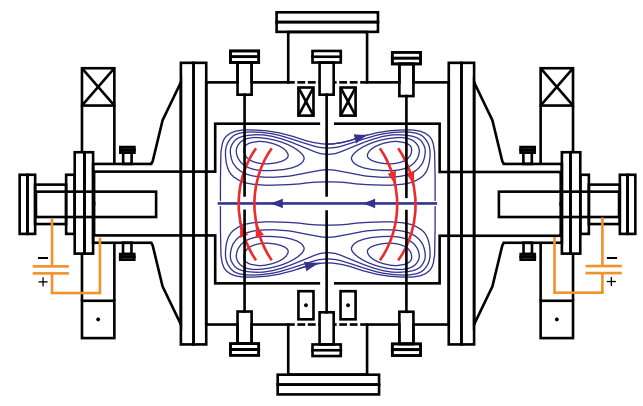

b)

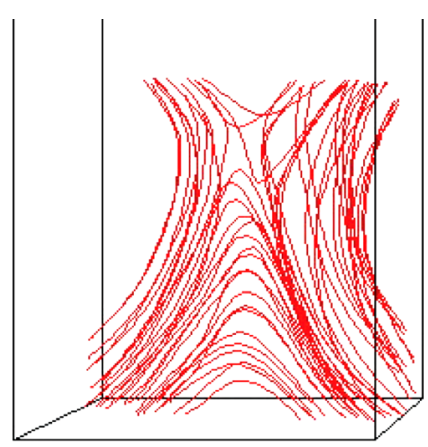

FIG. 1. Swarthmore Spheromak Experiment. The prolate flux conserver is $0.4 \mathrm{~m}$ in diameter and $0.6 \mathrm{~m}$ in length. (a) Schematic showing orientation of two merging spheromaks (with calculated flux surfaces) (b) magnetic field lines measured by the probe array. 


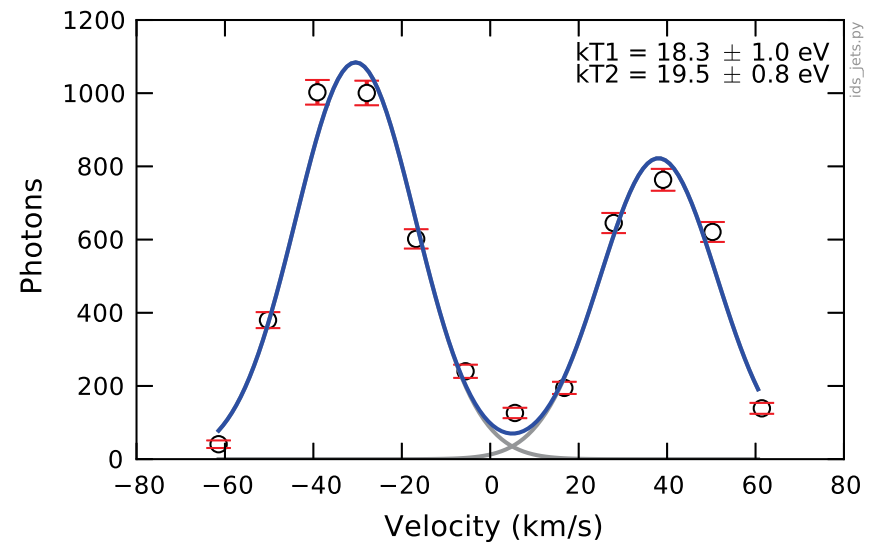

FIG. 2. Ion Doppler spectroscopy. Dynamics of $C_{I I I}$ line are depicted with fits to a double Gaussian model. Bi-directional jets are clearly evident at $40 \mu \mathrm{s}(-30 \mathrm{~km} / \mathrm{s}$ and $+38 \mathrm{~km} / \mathrm{s})$. Data are measured across a diameter at the midplane.

assumed constant throughout the reconnection zone, and (3) Alfvénic outflow. From these observations, we conclude from the density measurement and assumption of Alfvénic outflow that the magnetic field just at the inflow point of the reconnection layer is $B_{\text {in }}=440 \mathrm{G}$. This is consistent with local measurements but, moreover, this value defines the inflow boundary of the reconnection layer while the system is still close to axisymmetry. Since $E=v_{\text {out }} B_{\text {out }}$, we find that the magnetic field at the outflow boundary of the layer is $B_{\text {out }}=67 \mathrm{G}$. Since the inductive reconnection electric field is uniform in this steady-state analysis, we find $B_{\text {out }} / B_{\text {in }}$ $=v_{\text {in }} / v_{\text {out }}=\delta / L$. So we find for the aspect ratio $\delta / L$ $=0.15 \pm 0.02$. Again, this is consistent with internal measurements (see Figure 1) but determined with external, nonperturbative measurements.

At this stage, we can invoke a dissipation model to get absolute values for $L$ and $\delta$. If we assume a purely resistive model (Sweet-Parker) and Spitzer resistivity with $T_{e}$ $=15 \mathrm{eV},{ }^{19}$ we get a perpendicular diffusivity $14 \mathrm{~m}^{2} / \mathrm{s}$. We find $L=2 \mathrm{~cm}$ and $\delta=0.3 \mathrm{~cm}$ (this comes directly from $\delta / L=1 / \sqrt{S}$ with $\left.S=\mu_{0} v_{A} L / \eta\right)$. Using internal probes, we always measure larger values than this for the layer

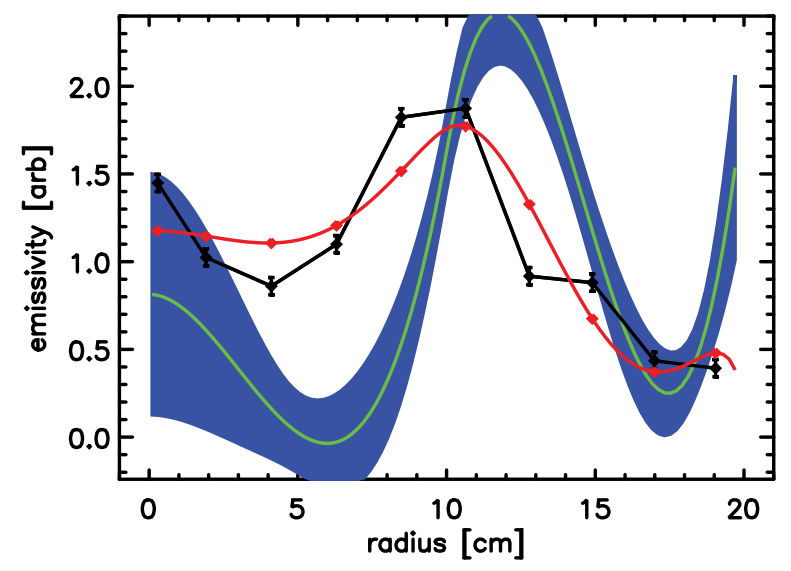

FIG. 3. IDS Abel inversion. Radial scan of total emissivity at $40 \mu$ s across 10 chords. A spline fit of measured emissivity (black curve with error bars) is Abel-inverted to generate a plot of the emissivity as a function of radius (blue curve with blue band for error bars). (a)

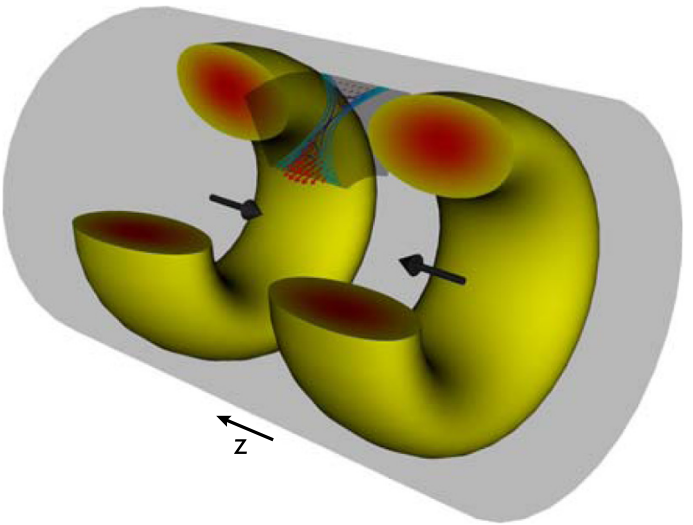

(b)

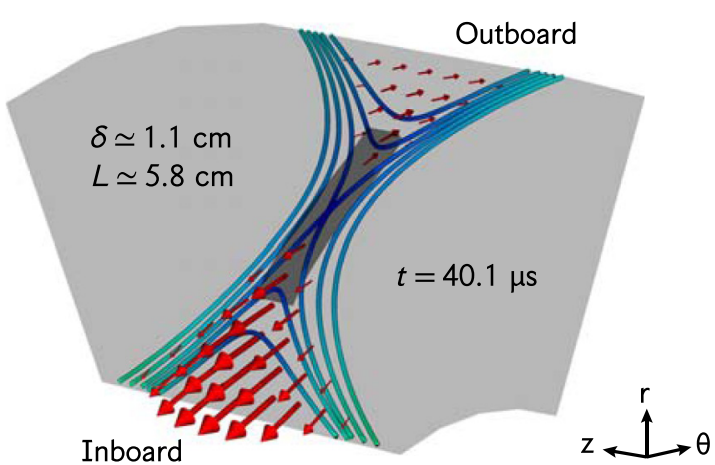

FIG. 4. Simulation results: (a) Global topology of the merging spheromaks and the reconnection layer that forms between them. (b) Closeup of the reconnection layer showing selected magnetic field lines (blue) and outflow vectors (red) as well as the location of the current sheet (thin shaded region)

dimensions (typically full-width half-maximum gives $L=5 \mathrm{~cm}$ and $\delta=1 \mathrm{~cm}$ ) indicating that non-resistive physics or geometric effects are at play. It is interesting to note that the 2D steady state Sweet-Parker like analysis provides useful guidance in modeling 3D dynamical plasmas like SSX, though this analysis predicts a smaller region than is measured.

Three-dimensional resistive MHD simulations from the HYM code have been used to study the spheromak merging process in SSX. These simulations, which are described in detail in Myers et al., ${ }^{20}$ are performed in a cylindrical geometry with a conducting boundary that matches the dimensions of the SSX flux conserver. High resolution meshes with 513 $\times 129 \times 32\left(N_{z} \times N_{r} \times N_{\theta}\right)$ nodes are employed to resolve the details of the reconnection current sheet that forms between the two merging spheromaks. The resulting simulations, which can be directly compared to experimental measurements, help to explain the novel merging behavior that is observed in the SSX experiments. ${ }^{20,21}$

Analysis of the reconnection layer in these simulations reveals several key features (see Fig. 4 compared to Fig. 1). First, the layer is obliquely inclined in the $r$ - $\theta$ plane because both poloidal and toroidal magnetic field components are reconnecting. This inclined current sheet drives outflows in both the $r$ and $\theta$ directions, though the bulk of the outflow is toroidally directed $\left(v_{\theta}>v_{r}\right)$. Second, the toroidal geometry of the merging plasmas produces different outflows on the inboard and outboard sides of the current sheet. In particular, faster outflows are observed on the inboard side, where the 

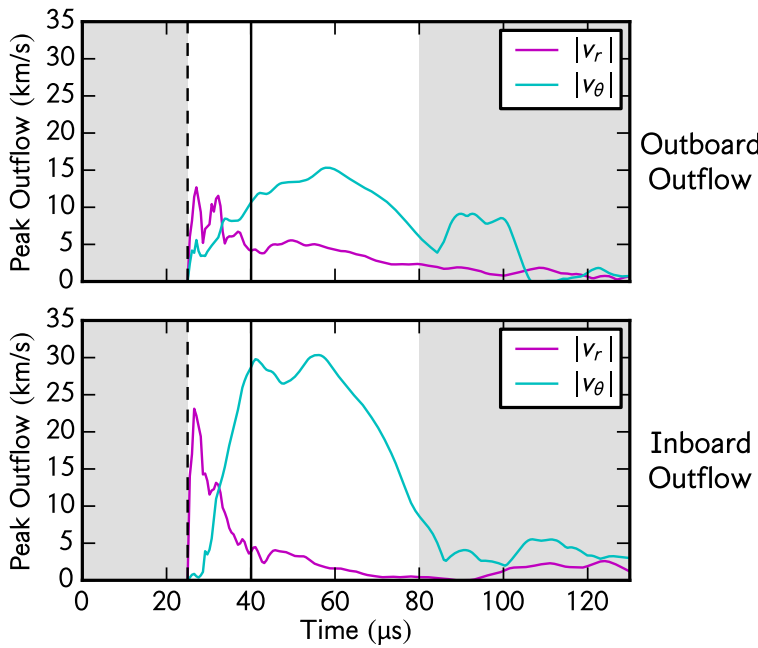

FIG. 5. Quantitative analysis of reconnection outflows in the simulations. Note that the toroidal geometry of the plasma causes the outflows on the outboard side of the sheet (top plot) to be slower than the outflows on the inboard side (bottom plot). In both regions, however, the outflows are predominantly toroidal $\left(v_{\theta}>v_{r}\right)$ and slowly evolving compared to the transient radial jets observed in the experiments.

cross section of the outflow channel is compressed by the toroidal geometry. These studies also demonstrated that the closest fit to experimental data featured an initial large-scale Lundquist number and Reynolds number both about 700 and that plasma viscosity can be responsible for slow reconnection of toroidal field and slower-than-ideal growth of the $n=1$ mode in SSX-like geometry. ${ }^{20}$ In both the simulation and the experiment, the reconnection dynamics drives the local Lundquist number in the layer to a much smaller value, $S_{\text {local }} \approx(L / \delta)^{2}$.

Quantitative analysis of the simulated reconnection layer facilitates direct comparisons to the experimental measurements. One useful comparison concerns the dimensions of the current sheet, which are defined by the fullwidth half-maximum (FWHM) of the reconnection current density profile. In particular, the width $\delta$ is determined from the axial FWHM, while the length $L$ is determined from the radial FWHM. This analysis shows that the simulated current sheet is virtually the same size as the current sheet measured in the SSX experiments: $\delta=1.1 \mathrm{~cm}, L=5.8 \mathrm{~cm}$. This agreement is interesting given the Sweet-Parker analysis above predicts a smaller region $(\delta=0.3 \mathrm{~cm}, L=2 \mathrm{~cm})$. It is also interesting that an MHD simulation accurately models a plasma in which the Larmor radius is comparable to the layer thickness $\left(\rho_{i} \sim \delta\right)$.

A second important comparison between the simulations and the experiments concerns the reconnection outflows, which are the focus of this paper. The outflows in the simulations are similar in magnitude to those observed in the experiment $(10-30 \mathrm{~km} / \mathrm{s})$, but they differ in two key ways: (1) the simulation outflows are primarily toroidal rather than radial and (2) the simulation outflows are slowly evolving rather than bursty (see Fig. 5). The bursty nature of the outflow events in the experiment are likely due to small nonaxisymmetries and slight axial shifts not captured in the simulation.

Reconnection in SSX is fully three-dimensional ${ }^{22}$ yet if we analyze the reconnection geometry shortly after merging, while the system is close to axisymmetry, the twodimensional Sweet-Parker like analysis, complete with nearly Alfvénic outflows, provides a useful interpretation of the experimental results. Here we report a precise, direct spectroscopic measurement of simultaneous bi-directional outflows from a reconnection event in a laboratory plasma. Abel inversion of emission data show that flows come predominantly from the core of the plasma where reconnection is known to take place. The external measurements of outflow speed $v_{\text {out }}$ as well as reconnection electric field and lineaveraged density allow us to infer details of the internal structure of the reconnection layer. The externally predicted aspect ratio $\delta / L$ agrees well with both internal measurements and a 3D simulation, though something other than steadystate $2 \mathrm{D}$ resistive physics is at play.

This work was supported by grants from the Department of Energy and the National Science Foundation (CMSO). The authors gratefully acknowledge the assistance of V. Lukin, D. Cohen, V. Chaplin, S. Palmer, and J. Haldeman.

${ }^{1}$ E. Priest and T. Forbes, Magnetic Reconnection (Cambridge University Press, Cambridge, 2000).

${ }^{2}$ T. Yokoyama, K. Akita, T. Morimoto, K. Inoue, and J. Newmark, Astrophys. J. 546, L69 (2001).

${ }^{3}$ D. E. Innes, B. Inhester, W. I. Axford, and K. Wilhelm, Nature 386, 811 (1997).

${ }^{4}$ D. E. Innes, P. Brekke, D. Germerott, and K. Wilhelm, Sol. Phys. 175, 341 (1997).

${ }^{5}$ K. Shibata, S. Masuda, M. Shimojo, H. Hara, T. Yokoyama, S. Tsuneta, T. Kosugi, and Y. Ogawara, Astrophys. J. 451, L83 (1995).

${ }^{6}$ T. Yokoyama and K. Shibata, Nature 375, 42 (1995).

${ }^{7}$ J. W. Cirtain et al., Science 318, 1580 (2007).

${ }^{8}$ T. D. Phan et al., Nature 404, 848 (2000).

${ }^{9}$ P. M. Nilson et al., Phys. Rev. Lett. 97, 255001 (2006)

${ }^{10}$ P. M. Nilson et al., Phys. Plasmas 15, 092701 (2008).

${ }^{11}$ S. Hsu, G. Fiksel, T. A. Carter, H. Ji, R. M. Kulsrud, and M. Yamada, Phys. Rev. Lett. 84, 3859 (2000).

${ }^{12}$ A. Frank, N. Kryie, and S. Satunin, Phys. Plasmas 18, 111209 (2011).

${ }^{13}$ W. Gekelman, R. L. Stenzel, and N. Wild, J. Geophys. Res. 87(A1), 101, doi:10.1029/JA087iA01p00101 (1982).

${ }^{14}$ C. G. R. Geddes, T. W. Kornack, and M. R. Brown, Phys. Plasmas 5, 1027 (1998).

${ }^{15}$ M. R. Brown, Phys. Plasmas 6, 1717 (1999).

${ }^{16}$ M. Landreman, C. D. Cothran, M. R. Brown, M. Kostora, and J. T. Slough, Rev. Sci. Instrum. 74, 2361 (2003).

${ }^{17}$ C. D. Cothran, J. Fung, M. R. Brown, and M. J. Schaffer, Rev. Sci. Instrum. 77, 063504 (2006).

${ }^{18}$ C. D. Cothran, A. Falk, A. Fefferman, M. Landreman, M. R. Brown, and M. J. Schaffer, Phys. Plasmas 10, 1748 (2003).

${ }^{19}$ V. H. Chaplin, M. R. Brown, D. H. Cohen, T. Gray, and C. D. Cothran, Phys. Plasmas 16, 042505 (2009).

${ }^{20}$ C. Myers, E. V. Belova, M. R. Brown, T. Gray, C. D. Cothran, and M. J. Schaffer, Phys. Plasmas 18, 112512 (2011).

${ }^{21}$ E. V. Belova, R. C. Davidson, H. Ji, M. Yamada, C. D. Cothran, M. R. Brown, and M. J. Schaffer, Nucl. Fusion 46, 162 (2006).

${ }^{22}$ C. D. Cothran, M. Landreman, W. H. Matthaeus, and M. R. Brown, Geophys. Res. Lett. 30, 1213, doi:10.1029/2002GL016497 (2003). 